\title{
ON $\left(L^{p_{0}}\left(A_{0}\right), L^{p_{1}}\left(A_{1}\right)\right)_{\theta, q}$
}

\author{
MICHAEL CWIKEL
}

Abstract. The Lions-Peetre formula for $\left(L^{p_{0}}\left(A_{0}\right), L^{p_{1}}\left(A_{1}\right)\right)_{\theta, q}$ valid for $q=p(\theta)$, where $1 / p(\theta)=(1-\theta) / p_{0}+\theta / p_{1}$, is shown to have no reasonable generalization for any $q \neq p(\theta)$.

Let $B$ be a Banach space, and $(X, \Sigma, \mu)$ a measure space. For $1 \leqq p \leqq \infty$, $L_{X}^{p}(B)$ is defined as the Banach space of strongly measurable $B$-valued functions $f$ on $X$, for which $\|f(x)\|_{B}$ belongs to the corresponding $L^{p}$ space $L_{X}^{D}$ of real valued functions. The subscript $X$ is omitted where this would not cause any ambiguity. We also take $X^{2}$ to denote the measure space obtained by a cartesian product of $X$ with itself, equipped with product measure, and $R_{+}$to denote $(0, \infty)$ equipped with Lebesgue measure.

Let $\left(A_{0}, A_{1}, \mathscr{A}\right)$ be an interpolation triple. It was shown in [3] that for $p_{0}, p_{1}$ in $[1, \infty]$ and $0<\theta<1$,

$$
\left(L^{p_{0}}\left(A_{0}\right), L^{p_{1}}\left(A_{1}\right)\right)_{\theta, q}=L^{q}\left(\left(A_{0}, A_{1}\right)_{\theta, q}\right)
$$

provided that $q=p(\theta)$, where $1 / p(\theta)=(1-\theta) / p_{0}+\theta / p_{1}$. The problem of identifying $\left(L^{p_{0}}\left(A_{0}\right), L^{p_{1}}\left(A_{1}\right)\right)_{\theta, q}$ for other values of $q$ was left open. In the special case where $A_{0}=A_{1},\left(L^{p_{0}}\left(A_{0}\right), L^{p_{1}}\left(A_{1}\right)\right)_{\theta, q}=L(p(\theta), q)\left(A_{0}\right)$, the space of strongly measurable $A_{0}$-valued functions $f$ such that $\|f(x)\|_{A_{0}}$ belongs to $L(p(\theta), q)$. (For definitions and details concerning the Lorentz spaces $L(p, q)$, see [1], [2], [4].) This example, as well as the LionsPeetre formula, suggests that, given $p_{0}, p_{1}, \theta$ and $q$, it might be possible to find a normed space $A, A \subset A_{0}+A_{1}$, such that the membership of $f(x)$ in $\left(L^{p_{0}}\left(A_{0}\right), L^{p_{1}}\left(A_{1}\right)\right)_{\theta, q}$ is determined solely by the behaviour of the real valued function $\|f(x)\|_{A}$.

What is such a space $A$ likely to be? A rather natural guess would be $A=\left(A_{0}, A_{1}\right)_{\theta, a}$, and indeed, if the measure space consists of a single atom of finite measure,

$$
\begin{aligned}
\left(L^{p_{0}}\left(A_{0}\right), L^{p_{1}}\left(A_{1}\right)\right)_{\theta, q}=\left(A_{0}, A_{1}\right)_{\theta, q} \\
\quad=\left\{f(x), \text { strongly }\left(A_{0}+A_{1}\right) \text { measurable: }\|f(x)\|_{\left(A_{0}, A_{1}\right)_{\theta, q}}<\infty\right\} .
\end{aligned}
$$

Received by the editors July 9, 1973.

AMS (MOS) subject classifications (1970). Primary 46E30, 46E35, 46E40.

Key words and phrases. Interpolation spaces, vector-valued $L^{p}$ space, $L(p, q)$ space.

(c) American Mathematical Society 1974 
From this it follows that in fact $\left(A_{0}, A_{1}\right)_{\theta, q}$ is the only choice for $A$ open to us (unless we envisage an $A$ depending on the structure of the underlying measure space).

In effect we are asking whether there exists a representation in the style of the Lions-Peetre formula:

$$
\begin{aligned}
\left(L^{p_{0}}\left(A_{0}\right), L^{p_{1}}\left(A_{1}\right)\right)_{\theta, q} \\
\quad=\left\{f(x), \text { strongly }\left(A_{0}+A_{1}\right) \text {-measurable: }\|f(x)\|_{\left(A_{0}, A_{1}\right)_{\theta, q}} \in S\right\}
\end{aligned}
$$

where $S$ is some class of real valued functions on $X$, depending on some or maybe all of $p_{0}, p_{1}, \theta, q, X, \Sigma, \mu, A_{0}, A_{1}$, and $\mathscr{A}$. We note in passing that for $S=L(p(\theta), q)$ the formula (1) is true in each of the three special cases, $q=p(\theta), A_{0}=A_{1}$ (provided $p_{0} \neq p_{1}$ ) and $X=$ single atom. Nevertheless, as we shall show here, $(1)$ is false for at least one choice of $\left(A_{0}, A_{1}, \mathscr{A}\right)$ and $(X, \Sigma, \mu)$ and for "nearly" every choice of parameters, $p_{0}, p_{1}, \theta, q$.

THEOREM. For each choice of parameters $\theta \in(0,1), p_{0}, p_{1}, q \in[1, \infty]$ with $p_{0} \neq p_{1}$ and $q \neq p(\theta)$ there exist $\left(A_{0}, A_{1}, \mathscr{A}\right)$ and $(X, \Sigma, \mu)$ and two strongly $\left(A_{0}+A_{1}\right)$ measurable functions $f$ and $g$ on $X$ such that,

$$
\begin{gathered}
f \in\left(L^{p_{0}}\left(A_{0}\right), L^{p_{1}}\left(A_{1}\right)\right)_{\theta, q}, \quad g \notin\left(L^{p_{0}}\left(A_{0}\right), L^{p_{1}}\left(A_{1}\right)\right)_{\theta, q}, \\
\|f(x)\|_{\left(A_{0}, A_{1}\right)_{\theta, q}}=\|g(x)\|_{\left(A_{0}, A_{1}\right)_{\theta, q}} \text { for all } x \in X .
\end{gathered}
$$

Proof. We take $A_{0}=L_{X}^{p_{0}}, A_{1}=L_{X}^{p_{1}}$ and $X=R_{+}$. Since $p_{0} \neq p_{1}$, $\left(A_{0}+A_{1}\right)_{\theta, q}=L_{X}(p(\theta), q)$ and

$$
\left(L^{p_{0}}\left(A_{0}\right), L^{p_{1}}\left(A_{1}\right)\right)_{\theta, q}=\left(L_{X^{2}}^{p_{0}}, L_{X^{2}}^{p_{1}}\right)_{\theta, q}=L_{X^{2}}(p(\theta), q) .
$$

Let $L_{X}(p, q)\left(L_{X}(p, q)\right)$ be the space of functions $f(x, y)$, measurable on $X^{2}$ such that $\|f(x, \cdot)\|_{L_{X}(p, q)} \in L_{X}(p, q)$ as a function of $x$.

LEMMA. If $p \neq q$,

$$
L_{R_{+}^{2}}(p, q) \neq L_{R_{+}}(p, q)\left(L_{R_{+}}(p, q)\right),
$$

and neither space contains the other.

The Lemma is proved in a later section. Take $p=p(\theta)$. Let $g(x, y)$ be a function on $X^{2}$ which belongs to $L_{X}(p, q)\left(L_{X}(p, q)\right)$ but not to $L_{X^{2}}(p, q)$. As a function of $x, G(x)=\|g(x, \cdot)\|_{L_{X}(p, q)}$ must belong to $L_{X}(p, q)$. Let $E$ be a subset of $X$ such that $\left\|\chi_{E}\right\|_{L_{X}(p, q)}=1$ and let $f(x, y)=G(x) \chi_{E}(y)$. Then we have that

$$
f(x, y) \in L_{X^{2}}(p, q) \text { and } g(x, y) \notin L_{X^{2}}(p, q),
$$


but also that

$$
\|f(x, \cdot)\|_{L_{X}(p, q)}=\|g(x, \cdot)\|_{L_{X}(p, q)} \text { for all } x .
$$

This completes the proof.

REMARK. It follows immediately that a formula of form (1) can never be generally true if $q \neq p(\theta)$ when $p_{0}$ and $p_{1}$ are unequal. However one might still hope that in the case $p_{0}=p_{1}$, where the above set of counterexamples is inapplicable, there exists a generalization of the LionsPeetre formula of form (1) for $q \neq p(\theta)$. Again we show that this is not so. Put $p=p_{0}=p_{1}=p(\theta)$. Then if $A_{0}=A_{1}$, (1) is true with $S=L_{X}^{p}$ for all values of $\theta$ and $q$. So unless we were to admit an $S$ which is allowed to vary its form depending on whether $A_{0}$ and $A_{1}$ are equal or not, the only possible version of (1) would be with $S=L_{X}^{p}$.

In fact if $q \leqq p$,

$$
\left(L^{p}\left(A_{0}\right), L^{p}\left(A_{1}\right)\right)_{\theta, q} \subset L^{p}\left(\left(A_{0}, A_{1}\right)_{\theta, q}\right)
$$

and the reverse inclusion holds for $q \geqq p$. These are readily proved using the integral version of Minkowski's inequality, and the observation that $f(x) \in\left(L^{p}\left(A_{0}\right), L^{p}\left(A_{1}\right)\right)_{\theta, q}$ if and only if

$$
\int_{0}^{\infty} t^{-\theta q}\left(\int_{X} K(t, f(x))^{p} d \mu\right)^{a / p} \frac{d t}{t}<\infty .
$$

[Here we adopt the standard notation

for each $x$.]

$$
K(t, f(x))=K\left(t, f(x), A_{0}, A_{1}\right)=\inf _{g+h=f(x)}\left(\|g\|_{A_{0}}+t\|h\|_{A_{1}}\right)
$$

To show that these inclusions are not, in general, equalities consider for example the case $q=\infty$. Here

$$
L^{p}\left(\left(A_{0}, A_{1}\right)_{\theta, \infty}\right) \subset\left(L^{p}\left(A_{0}\right), L^{p}\left(A_{1}\right)\right)_{\theta, \infty} .
$$

Let $X=R_{+}$, and $A_{0}=L_{R_{+}}^{1}, A_{1}=L_{R_{+}}^{\infty}$. Then $K(t, f)=\int_{0}^{t} f^{*}(s) d s$ (see $\left[1\right.$, p. 184]). Let $f(x, \cdot)=x^{-(2-\theta) / p} \chi_{\left(0, x^{1 / p}\right]}(\cdot)$. Then

$$
\begin{aligned}
K(t, f(x)) & =t x^{-(2-\theta) / p} & & \text { for } 0<t \leqq x^{1 / p} \\
& =x^{-(1-\theta) / p} & & \text { for } x^{1 / p} \leqq t<\infty
\end{aligned}
$$

So $\|f\|_{\left(A_{0}, A_{1}\right)_{\theta, \infty}}=\sup _{t>0} t^{-\theta} K(t, f)=x^{-1 / p} \notin L^{p}$.

However

$$
\begin{aligned}
\int_{X} K(t, f(x))^{p} d \mu & =\int_{0}^{t^{p}} x^{-(1-\theta)} d x+t^{p} \int_{t^{p}}^{\infty} x^{-(2-\theta)} d x \\
& =(1 / \theta+1 /(1-\theta)) t^{\theta p}
\end{aligned}
$$


so

$$
\sup _{t>0} t^{-\theta}\left(\int_{X} K(t, f(x))^{p} d \mu\right)^{1 / p}<\infty \text { and } f \in\left(L^{p}\left(A_{0}\right), L^{p}\left(A_{1}\right)\right)_{\theta, \infty},
$$

proving that (2) is strict. We could equally well have used

$$
g(x, \cdot)=e^{-x(1-\theta)} \chi_{\left(0, e^{x}\right]}(\cdot)
$$

on $(-\infty, \infty)$ instead of $f(x, \cdot)$ on $R_{+} \cdot\|g(x, \cdot)\|_{\left(A_{0}, A_{1}\right)_{\theta . \infty}}=1$ for all $x$, and as we have already noted $\|f(x, \cdot)\|_{\left(A_{0}, A_{1}\right)_{\theta, \infty}}=x^{-1 / p^{\infty}}$. Thus we may also remark that

$$
\left(L^{p}\left(A_{0}\right), L^{p}\left(A_{1}\right)\right)_{\theta, \infty} \notin L(q, r)\left(\left(A_{0}, A_{1}\right)_{\theta, \infty}\right)
$$

for all $q$ and $r$.

Proof of THE Lemma. We show that neither of the spaces $A=$ $L_{R_{+}^{2}}(p, q)$ and $B=L_{R_{+}}(p, q)\left(L_{R_{+}}(p, q)\right)$ contains the other if $p \neq q$. It is convenient to use the natural quasinorms for the spaces $A$ and $B$ (see [2], [4]),

$$
\begin{aligned}
\|f\|_{A}^{*}=\|f\|_{p, q}^{*} & =\left(\int_{0}^{\infty} t^{q / p-1} f^{*}(t)^{q} d t\right)^{1 / q}, & & q<\infty, \\
& =\sup _{t>0} t^{1 / p} f^{*}(t), & & q=\infty,
\end{aligned}
$$

where $f^{*}(t)$ is the nonincreasing rearrangement on $R_{+}$of the function $f(x, y)$ on $R_{+}^{2}$.

$$
\|f\|_{B}^{*}=\left\|\left(\|f(x, \cdot)\|_{p, q}^{*}\right)\right\|_{p, q}^{*},
$$

where here $\|\cdot\|_{p, q}^{*}$ denotes the usual $L_{R_{+}}(p, q)$ quasinorm. It is first evaluated for $f(x, y)$ as a function of $y$ for each $x$, and subsequently for the resulting function of $x$.

(i) Consider $q=\infty . g(x, y)=1 /(x y)^{1 / p}$ is in $B$ but not $A$. For the reverse noninclusion, define

$$
\begin{array}{rlrl}
f(x, y) & =0 & \text { for } y>2 x^{-3} \exp \left(-1 / x^{2}\right) \\
& =\exp \left(1 / p x^{2}\right) \quad \text { for } 0 \leqq y \leqq 2 x^{-3} \exp \left(-1 / x^{2}\right) . \\
\|f\|_{A}^{*} & =\sup _{t>0} t^{1 / p} f^{*}(t) \\
& =\sup _{s>0}\left(\int_{0}^{s} 2 x^{-3} \exp \left(-1 / x^{2}\right) d x\right)^{1 / p} \exp \left(1 / p s^{2}\right) \\
& =\sup _{s>0}\left(\exp \left(-1 / s^{2}\right)\right)^{1 / p} \exp \left(1 / p s^{2}\right)=1 .
\end{array}
$$

But

$$
\begin{aligned}
\|f(x, \cdot)\|_{p, \infty}^{*} & =\left[2 x^{-3} \exp \left(-1 / x^{2}\right)\right]^{1 / p} \exp \left(1 / p x^{2}\right) \\
& =\left[2 x^{-3}\right]^{1 / p} .
\end{aligned}
$$


Therefore

$$
\|f\|_{B}^{*}=\sup _{x>0} x^{1 / p}\left[2 x^{-3}\right]^{1 / p}=\infty .
$$

(ii) $q<\infty$. Each counterexample function will be constructed similarly to $f$ above. Take a nonnegative function $e(x)$ and a nonnegative nonincreasing continuous function $F(x)$ and define

$$
\begin{aligned}
f(x, y) & =0 & & \text { for } y>e(x), \\
& =F(x) & & \text { for } 0 \leqq y \leqq e(x) .
\end{aligned}
$$

Then, if $E(x)=\int_{0}^{x} e(t) d t$,

and

$$
\begin{aligned}
\|f\|_{A}^{*} & =\left(\int_{0}^{\infty} t^{\alpha / p-1} f^{*}(t)^{q} d t\right)^{1 / \alpha} \\
& =\left(\int_{0}^{\infty} E(x)^{q / p-1} F(x)^{q} e(x) d x\right)^{1 / \alpha}
\end{aligned}
$$

$$
\begin{aligned}
\|f(x, \cdot)\|_{p, q}^{*} & =F(x)\left(\int_{0}^{e(x)} t^{q / p-1} d t\right)^{1 / q} \\
& =(p / q)^{1 / q} e(x)^{1 / p} F(x) .
\end{aligned}
$$

In each of the following cases $e$ and $F$ will be chosen to ensure also that $e(x)^{1 / p} F(x)$ is nonincreasing and continuous. Then

$$
\|f\|_{B}^{*}=(p / q)^{1 / q}\left(\int_{0}^{\infty} x^{q / p-1} F(x)^{q} e(x)^{q / p} d x\right)^{1 / q} .
$$

First suppose $p<q$. Put

so

$$
e(x)=\exp x \cdot \exp (\exp x)
$$

Put

$$
E(x)=\exp (\exp x)-\exp 1 \text {. }
$$

$$
\begin{aligned}
F(x) & =1 / e(x)^{1 / p} . \\
\|f\|_{A}^{* a} & =\int_{0}^{\infty}[E(x) / e(x)]^{\alpha / p-1} d x \\
& \leqq \int_{0}^{\infty}[\exp (-x)]^{\alpha / p-1} d x<\infty .
\end{aligned}
$$

But $\|f\|_{B}^{* a}=(p / q) \int_{0}^{\infty} x^{q / p-1} d x=\infty$. Thus $A \notin B$.

Next put $e(x)=1 /(x+1)$, so

$$
\begin{gathered}
E(x)=\log (x+1) \text { and } F(x)=\min \left\{1,[\log (x+1)]^{-1 / p}\right\} . \\
\|f\|_{A}^{* a} \geqq \int_{e-1}^{\infty}[\log (x+1)]^{q / p-1-q / p}(x+1)^{-1} d x=\infty
\end{gathered}
$$


But

$$
\|f\|_{B}^{* q} \sim(p / q) \int_{e-1}^{\infty} x^{q / p-1}[\log (x+1)]^{-q / p}(x+1)^{-q / p} d x<\infty
$$

proving $B \notin A$.

It remains to consider the case $p>q$. Again take $e(x)=1 /(x+1)$ so $E(x)=\log (x+1)$. Let

$$
\begin{aligned}
& F(x)=[\log (x+1)]^{-1 / p+\varepsilon / q} \text { for } 0 \leqq x \leqq e-1, \\
& =[\log (x+1)]^{-1 / p-\varepsilon / q} \text { for } x \geqq e-1 \text {, }
\end{aligned}
$$

with $0<\varepsilon<\min [q \mid p, 1-q / p]$.

$$
\begin{aligned}
\|f\|_{A}^{* \alpha}= & \int_{0}^{e-1}[\log (x+1)]^{\alpha / p-1}[\log (x+1)]^{-q / p+\varepsilon}(x+1)^{-1} d x \\
& +\int_{e-1}^{\infty}[\log (x+1)]^{q / p-1}[\log (x+1)]^{-q / p-\varepsilon}(x+1)^{-1} d x \\
= & \int_{0}^{1} r^{-1+\varepsilon} d r+\int_{1}^{\infty} r^{-1-\varepsilon} d r<\infty . \\
\|f\|_{B}^{* q} \geqq & (p / q) \int_{e-1}^{\infty}(x+1)^{q / p-1}[\log (x+1)]^{-q / p-\varepsilon}(x+1)^{-\alpha / p} d x \\
= & (p / q) \int_{1}^{\infty} r^{-q / p-\varepsilon} d r=\infty .
\end{aligned}
$$

Therefore $A \notin B$.

Finally put $e(x)=\exp x$, so $E(x)=\exp x-1$. Let

$$
\begin{aligned}
F(x) & =\exp (-x / p) & & \text { on }[0,1], \\
& =\exp (-x / p) x^{-1 / p-\varepsilon / q} & & \text { on }[1, \infty),
\end{aligned}
$$

where $0<\varepsilon<1-q / p$. Note that, as always $e(x)^{1 / p} F(x)$ is nonincreasing.

But

$$
\begin{aligned}
\|f\|_{A}^{* q} & \geqq \int_{1}^{\infty}(\exp x-1)^{q / p-1} x^{-q / p-\varepsilon}(\exp x)^{-q / p+1} d x \\
& \geqq \int_{1}^{\infty} x^{-q / p-\varepsilon} d x=\infty
\end{aligned}
$$

$$
\begin{aligned}
\|f\|_{B}^{* q}= & (p / q) \int_{0}^{1} x^{q / p-1} \exp (-q x / p) \exp (q x / p) d x \\
& +(p / q) \int_{1}^{\infty} x^{q / p-1} x^{-q / p-\varepsilon} d x \\
& <\infty
\end{aligned}
$$

showing that $B \nsubseteq A$ and completing the proof of the Lemma. 
REMARK. In fact the Lions-Peetre formula for $\left(L^{p_{0}}\left(A_{0}\right), L^{p_{1}}\left(A_{1}\right)\right)_{\theta, p(\theta)}$ is true for $p_{0}$ and $p_{1}$ in the extended range $(0, \infty]$ (see [4]). Similarly the Theorem and Lemma presented above remain valid for $p_{0}, p_{1}, q \in$ $(0, \infty]$.

ACKNowledgement. I would like to thank Dr. Yoram Sagher for his helpful comments and constant interest.

\section{REFERENCES}

1. P. L. Butzer and H. Berens, Semi-groups of operators and approximation, Die Grundlehren der math. Wissenschaften, Band 145, Springer-Verlag, New York, 1967. MR 37 \#5588.

2. R. A. Hunt, On $L(p, q)$ spaces, Enseignement Math. (2) 12 (1966), 249-276. MR 36 \#6921.

3. J. L. Lions and J. Peetre, Sur une classe d'espaces d'interpolation, Inst. Hautes Etudes Sci. Publ. Math. No. 19 (1964), 5-68. MR 29 \#2627.

4. Y. Sagher, Interpolation of r-Banach spaces, Studia Math. 41 (1972), 45-70.

Department of Theoretical Mathematics, Weizmann Institute of Science, REHOVOT, ISRAEL

Current address: Mathématique (Bât. 425), Université de Paris-Sud, Orsay 91405, France 